\title{
Impacts of a lower limb exoskeleton robot on the muscle strength of tibialis anterior muscle in stroke patients
}

\author{
Jiyu Zhang ${ }^{1, *}$, Tian Wang ${ }^{2,}$, Qingyu Zhao ${ }^{2}$, and Shichao Liu ${ }^{3}$ \\ ${ }^{1}$ School of Instrumentation Science and Engineering, Harbin Institute of Technology, 92 West Dazhi Street, Harbin, China \\ ${ }^{2}$ Hangzhou RoboCT Technological Development Co., Ltd., 2B-7F No. 1326 Wenyi West Road, Hangzhou, China \\ ${ }^{3}$ Department of Electronics, Carleton University, 1125 Colonel By Drive, Ottawa, Canada
}

\begin{abstract}
This work aims to explore the impact of a proposed lower limb exoskeleton robot on the muscle strength of the tibialis anterior muscle in stroke patients. Firstly, 24 patients with stroke hemiplegia were divided into the robot group and the control group according to a random number table. Both groups received conventional rehabilitation treatments. Moreover, the robot group took the walking training with UG0210, a lower limb exoskeleton walking rehabilitation device developed by the Hangzhou RoboCT Technology Development Co., Ltd., once per day, 30 minutes per time, a total of 20 times of treatment. The control group took the conventional rehabilitation walking training, once per day, 30 mins per time, a total of 20 times of treatment. At the beginning of the trial, the manual muscle strength test (MMT) was used to assess the pre-trial muscle strength within the trial cycle. The efficacy of the two groups was compared. Results The muscle strength of the tibialis anterior muscle was higher than that without treatments in both groups $(\mathrm{P}<0.05)$. The curative effect of the robot group was better than that of the control group $(\mathrm{P}<0.05)$. Conclusions With the help of the designed lower limb exoskeleton robot, both tibialis anterior muscle strength and lower limb motor function of stroke patients were improved compared to the control group. The comparison shows the attractive potential and value of the robot assisted rehabilitation.
\end{abstract}

\section{Introduction}

Epidemiological survey showed that the the annual number of patients with stroke in China was more than 1.5 million, with an incidence rate as high as $217 / 100$, 000 and a disability rate of $86.5 \%$ [1]. The foot drop is one of the common walking dysfunctions after the onset of the disease. When the patient steps forward with the lower limb, the pelvis will be compensatory elevated. At the same time, it will be accompanied by abduction and external rotation of the hip joint, forming a "circling gait" [2]. In order to restore and strengthen the muscle group of ankle dorsiflexion, the tibialis anterior muscle is usually stimulated by manipulation and various physical factors, to better reconstruct the walking ability. In recent years, with the in-depth and rapid development of the combination of medical field and industrial field, the exoskeleton robot has attracted wide attention to in the field of rehabilitation, and gradually is applied to the lower limb rehabilitation training. In this paper, the impact of the lower limb exoskeleton robot on the muscle strength of the anterior tibial muscle in stroke patients was analyzed.

\section{General data}

From October 2018 to January 2019, 24 patients with stroke hemiplegia and lower limb motor dysfunction were admitted to the Heilongiiang General Hospital of Agricultural Reclamation. After screening, the patients were randomly divided into two groups with 12 patents in each group. The general data were comparable between the two groups with no significant difference, as shown in Table 1. This study was approved by the medical ethics committee of the General Hospital of the State Bureau of Agricultural Reclamation.

\section{Inclusion criteria and exclusion criteria}

Inclusion criteria: All of them met the diagnostic criteria for stroke established by the 4th National Conference on Cerebrovascular Diseases [3]. The course of disease is 0 to 6 months. After treatment, the vital signs were stable, the condition was stable or improved. The patient was conscious and could follow the instructions, and cooperated well during treatment. Exclusion criteria: Patients with other neuromuscular and osteoarticular diseases affecting walking ability[4], combined with severe primary diseases such as cardiovascular, lung, liver, kidney, hematopoietic system and serious cognitive, hearing and vision disorders, who were participating in other clinical studies or were unwilling to participate in the study were excluded.

\footnotetext{
\rceil$^{\dagger}$ Corresponding author: wangtian@roboct.com
} 
Table 1. Comparison of demographic data between two groups.

\begin{tabular}{|c|c|c|c|c|}
\hline Project & Robot group & Control group & Statistic & $\mathrm{p}$ value \\
\hline \multicolumn{5}{|l|}{ Age (year) } \\
\hline N(Missing) & 12 & 12 & \multirow{6}{*}{$0.52(\mathrm{t})$} & \multirow{6}{*}{0.6151} \\
\hline Mean & 52.19 & 49.84 & & \\
\hline SD & 10.38 & 11.82 & & \\
\hline $\operatorname{Min} \sim \operatorname{Max}$ & $28.0 \sim 68.5$ & $27.5 \sim 65.5$ & & \\
\hline Median & 55.20 & 50.25 & & \\
\hline Shapiro-Wilk(P) & $0.92(0.2969)$ & $0.96(0.7950)$ & & \\
\hline \multicolumn{5}{|l|}{ Gender } \\
\hline Woman & $1(4.17 \%)$ & $3(12.50 \%)$ & \multirow{3}{*}{ (fisher) } & \multirow{3}{*}{0.5901} \\
\hline Man & $11(45.83 \%)$ & $9(37.50 \%)$ & & \\
\hline Amount & $12(50.00 \%)$ & $12(50.00 \%)$ & & \\
\hline \multicolumn{5}{|c|}{ Course of disease (month) } \\
\hline N(Missing) & 12 & 12 & \multirow{6}{*}{$1.40(\mathrm{t})$} & \multirow{6}{*}{0.1894} \\
\hline Mean & 2.88 & 1.96 & & \\
\hline $\mathrm{SD}$ & 2.17 & 0.73 & & \\
\hline $\operatorname{Min} \sim \operatorname{Max}$ & $0.5 \sim 7.6$ & $0.8 \sim 3.3$ & & \\
\hline Median & 2.20 & 1.85 & & \\
\hline Shapiro-Wilk(P) & $0.91(0.2086)$ & $0.94(0.5167)$ & & \\
\hline
\end{tabular}

Both groups took basic rehabilitation training, including trunk control training, hip and knee joint control training, ankle dorsiflexion induction training[5], once a day. On this basis, the two groups were given different types of walking training. The patients in the control group were given a 30 minutes routine walking training every day, including balance bar walking training, single leg weight-bearing training, up and down stairs training, etc. The patients in the experimental group were given a 30 minutes walking training every day with the lower limb exoskeleton walking rehabilitation device. Fig.1 shows the patient in experimental group rehabilitation walking training with lower limb exoskeleton robot. The patient could be allowed to deviate from the treatment for less than or equal to 2 times during continuous 20 times of training (except Sunday).

\section{Evaluation Method}

Evaluation method: Each patient was evaluated by the same therapist before the start of the trial and at the end of the whole trial cycle. And the muscle strength of tibialis anterior muscle was evaluated by using the manual muscle strength test (MMT).

\section{Statistical analysis}

SAS9.2 was used for statistical analysis, and SAS macro language was used to write programs and run programs to complete all statistical contents. At each follow-up point, the comparison between the two groups was performed by independent sample t-test. The comparison within the group before and after training was performed

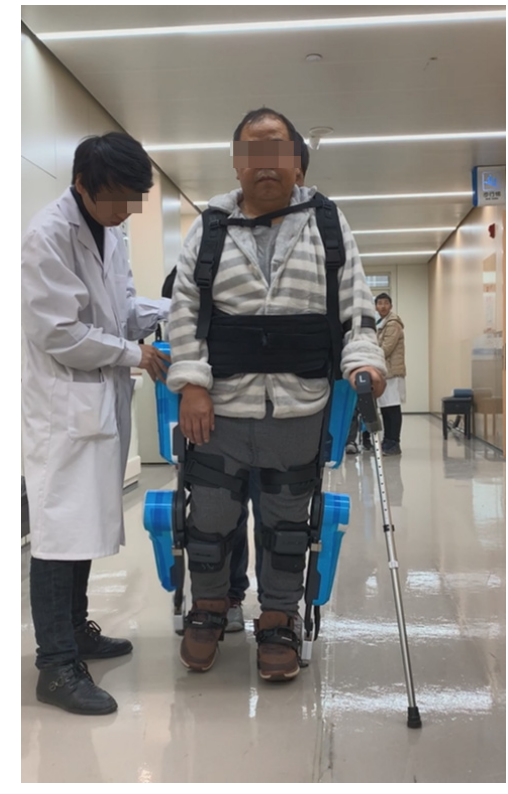

Fig. 1. The patient is training with lower limb exoskeleton robot. 
Table 2. Significance test of the difference of rating results in different stages between two groups.

\begin{tabular}{|c|c|c|c|}
\hline Stage & Cases(missing) & Statistics $(Z)$ & $\mathrm{p}$ value \\
\hline \multicolumn{4}{|c|}{ Without treatment } \\
\hline $\begin{array}{l}\text { Robot group } \\
\text { Control group }\end{array}$ & $\begin{array}{l}12(0) \\
12(0)\end{array}$ & \multirow[t]{2}{*}{0.67} & 0.5037 \\
\hline \multicolumn{3}{|c|}{ With treatment } & \\
\hline $\begin{array}{l}\text { Robot group } \\
\text { Control group }\end{array}$ & $\begin{array}{l}12(0) \\
11(1) \\
\end{array}$ & -1.31 & 0.1917 \\
\hline
\end{tabular}

Table 3. Paired test of rating results before and after treatment and comparison of differences between the two groups.

\begin{tabular}{cccccc}
\hline & & $\begin{array}{c}\text { Comparison before and after treatment } \\
\text { in group }\end{array}$ & \multicolumn{2}{c}{$\begin{array}{c}\text { Comparison before and after } \\
\text { treatment between groups }\end{array}$} \\
\hline & Cases & Statistic(S) & p value & Cases (Z) & p value \\
Robot group & 12 & 27.5 & 0.0020 & -2.73 & 0.0064 \\
Control group & 11 & 10.5 & 0.0313 & & \\
\hline
\end{tabular}

\section{Conclusion}

At the beginning of the trial, 12 patients were included in each group, while 1 patient dropped out in the control group at the late stage of treatment. The independent sample t-test showed that the $\mathrm{p}$ value was $>0.05$, there was no statistical difference, as shown in Table 2.

Paired t-test was used to compare within the groups before and after treatment. Both $\mathrm{p}$ values were less than 0.05 , with statistical difference. The muscle strength of the two groups was improved after treatment. Independent sample t-test was used for comparison between the groups before and after treatment. Both $\mathrm{p}$ values were less than 0.05 , with statistical difference. The robot group was superior to the control group, as shown in Table 3.

\section{Discussion}

After the central nervous system injury, the release of inhibited low central motor reflex is one of the main causes of foot ptosis[6]. And the tibialis anterior muscle is the main muscle group to control the ankle dorsiflexion. Some patients suffer from atrophy of tibialis anterior muscle due to being bedridden for a long time and having little movement of lower limbs. In addition, because of the corresponding abnormal increase of antagonistic muscle tension, the patients cannot touch the heel first at the moment of stepping. Clinically, the speed of nerve recovery is actually related to the different injury degrees and injury sites of the patient. Many people rush to go to the ground without the guidance of a therapist for a long time of walking training. In the common movement mode of extensor muscle formed after the central nerve system injury, the patient's foot dorsiflexion is often accompanied by foot varus, which increases the risk of falls. In the later stage, it is easy to lead to foot drop and foot varus, which is difficult to be corrected again[7,8].

A large number of studies have shown that early rehabilitation training can promote the reinnervation of the denervated tissues, and the compensation of the tissues around the focus can also contribute to the reconstruction of the neural feedback circuit[9]. In this experiment, compared with the conventional rehabilitation method, the use of the lower limb exoskeleton robot can simulate the correct gait repeatedly, which has more obvious advantages in improving the muscle strength of tibialis anterior muscle, improving the performance of ankle joint movement abnormality of patients, and further improving the walking ability of patients[10-12]. With the continuous intersection and integration of rehabilitation and various disciplines, the function of the lower limb exoskeleton robot will continue to be improved and updated, providing more training modes and methods for patients with different lower limb dysfunctions.

\section{Acknowledgement}

* This work was supported by the National Natural Science Foundation of China (No.51709068) and the Postdoctoral Foundation of Heilongjiang Province Government (No.LBH-Z17094).

\section{References}

1. X.D. Wang, Practical clinical neurology (Beijing, 2007)

2. X.P. Yun, Assessment of rehabilitation therapy (Beijing, 2005)

3. CNS, ChinJNeurol 26, 6 (1996)

4. Y.N. Zhang, Z.W. Hao, J.M. Li, S.H. Ma, H.T. Shen, C.X. Chen, Chinese Journal of Rehabilitation Medicine, 11, 27 (2012)

5. X.X. Feng, Y.H. Bai, Y.M. Xu, T.F. Fu, H. Zhang, D.M. Ye, G. Wang, Chinese Journal of Rehabilitation, 2 (2014)

6. K.L. Qian, T.Wang, Chinese Journal of Rehabilitation Medicine, 3, 16 (2001)

7. W.R. Zhao, H.H. Zhao, Chinese Journal of Rehabilitation Medicine, 12, 22 (2007) 
8. F. R, O. D, J. B, The Inter J of Clin Foot Science, 2, 19 (2002)

9. K.J. Sullivan, B.J. KnowIton, B.H. Dobkin, Arch Phys Med Rehabil, 83 (2002)

10. M. Bortole, A. Venkatakrishnan, F.S. Zhu, J.C. Moreno, G.E. Francisco, J.L. Pons, J.L. ContrerasVidal, JNER, 12, 1 (2015)

11. C. Buesing, G. Fisch, M, O 'Dorm, I. Shahidi, L. Thomas, C.K. Mummidisetty, K.J. Williams, H. Takahashi, W.Z. Rymer, Neuroeng Rehabil, 12, 1 (2015)

12. H. Fukuda, K. Samura, 0. Hamada, K. Saita., T. Ogata, E. Shiota, Y. Sankai, T. Inoue, Neurol Med Chir 55, 6 (2015) 